\title{
Individuelle und institutionelle Bedingungen gescheiterter Bildungs- investitionen: Bildungsarmut im internationalen Vergleich
}

In modernen wissensbasierten Ökonomien stellt der Anteil bildungsarmer Personen eine zunehmend wichtige sozialpolitische Kenngröße dar. Auf der Individualebene kann Bildungsarmut die Lebenschancen erheblich beeinträchtigen, auf der gesellschaftlichen Ebene drohen hohe soziale und politische Folgekosten. Im internationalen Vergleich zeigt sich, dass der Anteil bildungsarmer Jugendlicher stark zwischen verschiedenen Ländern variiert. Der Beitrag untersucht, wie Merkmale von Bildungssystemen die Unterschiede zwischen Ländern erklären können. Eine höhere Anzahl verschiedener Schulformen erhöht den Grad der Bildungsarmut, mehr Schulautonomie und nationale Bildungsstandards reduzieren sie.

JANNA TELTEMANN, MICHAEL WINDZIO

\section{Einleitung}

Bildung nimmt für den Zusammenhalt und den wirtschaftlichen Erfolg moderner Gesellschaften eine Schlüsselstellung ein. In Wissensgesellschaften sind Kompetenzen und Zeugnisse Voraussetzungen für Status und Teilhabe, und das $\mathrm{Hu}-$ mankapital der Bevölkerung stellt eine Grundlage für ökonomische Prosperität und sozialen Frieden dar. Somit kommen den Bildungssystemen moderner Gesellschaften zentrale gesellschaftliche Funktionen zu und ihr Erfolg zeigt sich unter anderem darin, in welchem Maße sie Bildungsarmut verhindern können. Bildungsarm ist, wer keinen Schulabschluss und keinen berufsqualifizierenden Ausbildungsabschluss hat oder dessen Lesekompetenzen am Ende der Pflichtschulzeit nicht ausreichen, um einfache Zusammenhänge in Texten zu erkennen. Von Bildungsarmut betroffenen Menschen drohen kumulative Benachteiligungen und Exklusionsrisiken (Bacher et al. 2010; Ludwig-Mayerhofer/Kühn 2010), auf gesellschaftlicher Ebene zieht Bildungsarmut zugleich hohe soziale und ökonomische Folgekosten nach sich (Allmendinger et al. 2011; Wößmann/Piopiunik 2009). Die intergenerationale Transmission von Bildungsarmut verstärkt diese Phänomene.

Die bisherige Forschung hat sich vor allem auf individuelle und gesellschaftliche Folgen von Bildungsarmut und damit auf die Verwertungsebene konzentriert (Solga 2011, S. 414; Solga 2005; Giesecke et al. 2010); weniger eindeutig sind bisher die Befunde zu den individuellen und den insti- tutionellen Ursachen von Bildungsarmut. Insbesondere für die Analyse von Bedingungsfaktoren auf der Ebene von Bildungssystemen eignen sich internationale Vergleiche, die die Untersuchung von Zusammenhängen zwischen variierenden Bildungssystemmerkmalen und dem Grad der Bildungsarmut ermöglichen. In unserem Beitrag möchten wir deshalb quantitative Befunde zu individuellen und institutionellen Einflüssen auf kompetenzbezogene Bildungsarmut darstellen. Im Folgenden erläutern wir das relativ neue Konzept der Bildungsarmut (Abschnitt 2) und stellen Theorien zur Erklärung von Bildungsarmut bzw. Bildungsungleichheit dar (3). Jutta Allmendinger (1989) folgend unterscheiden wir zwischen Stratifizierung und Standardisierung von Schulsystemen (4). Wir argumentieren aus einer handlungstheoretischen Perspektive, dass diese Dimensionen auch die Risiken der individuellen Bildungsarmut beeinflussen. In der empirischen Analyse werden die Ergebnisse von Mehrebenenregressionen mit Daten aus der OECD-PISA-Studie 2009 präsentiert (5 und 6). Abschnitt 7 resümiert die Befunde und skizziert offene Forschungsfragen.

\section{Was bedeutet Bildungsarmut?}

Bildungsarmut ist ein individuelles, jedoch institutionell geprägtes Merkmal, denn ihre Genese lässt sich zumin- 


\section{Kompetenzbezogene Bildungsarmut im Ländervergleich*}

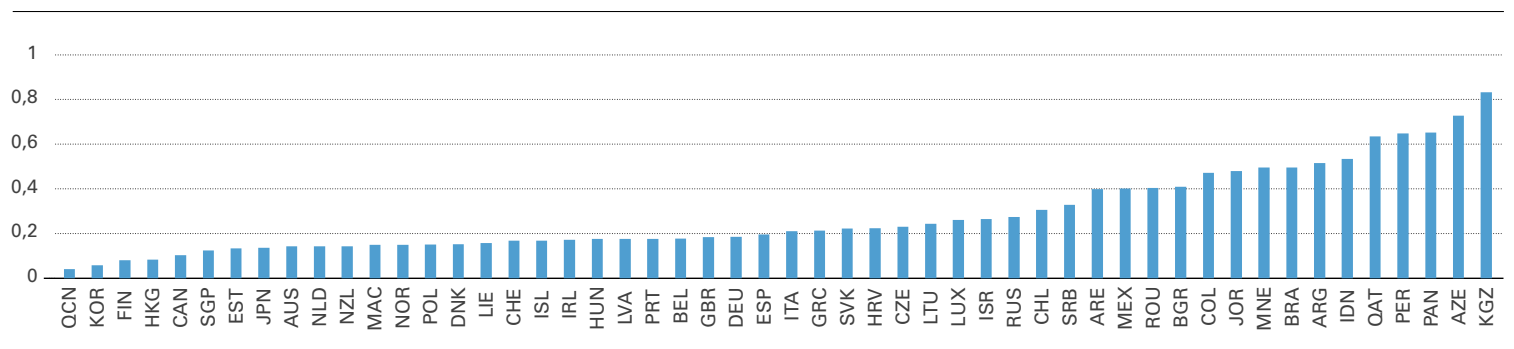

*Anteil der Schüler unter Kompetenzstufe 2 nach PISA-Kompetenzskala.

$\mathrm{ARE}=$ Vereinigte Arabische Emirate; $\mathrm{ARG}=$ Argentinien; $\mathrm{AUS}=$ Australien; $\mathrm{AZE}=$ Aserbaidschan; $\mathrm{BEL}=$ Belgien; $\mathrm{BGR}=$ Bulgarien; $\mathrm{BRA}=\mathrm{Brasilien} ; \mathrm{CAN}=\mathrm{Kanada}$; $\mathrm{CHE}=$ Schweiz; $\mathrm{CHL}=\mathrm{Chile} ; \mathrm{COL}=$ Kolumbien; $\mathrm{CZE}=$ Tschechien; $\mathrm{DEU}=$ Deutschland; $\mathrm{DNK}=$ Dänemark; $\mathrm{ESP}=\mathrm{Spanien} ; \mathrm{EST}=\mathrm{Estland} ; \mathrm{FIN}=\mathrm{Finnland}$;

$\mathrm{CHE}=$ Schweiz; $\mathrm{CHL}=\mathrm{Chil}, \mathrm{COL}=$ Kolumbien; $\mathrm{CZE}=$ Tschechien; $\mathrm{DEU}=$ Deutschland; $\mathrm{DNK}=$ Dănemark, ESP = Spanien; $\mathrm{EST}=\mathrm{EStland}$; $\mathrm{FIN}=\mathrm{Finnland}$;

$\mathrm{GBR}=$ Großbritannien; GRC = Griechenland; $\mathrm{HKG}=$ Hong Kong; $\mathrm{HRV}=$ Kroatien; $\mathrm{HUN}=$ Ungarn; IDN = Indonesien; IRL = Irland; ISL = Island; ISR = Israel; ITA = Italien;
JOR = Jordanien; JPN = Japan; KGZ = Kirgisistan; KOR = Korea; LIE = Liechtenstein; LTU = Litauen; LUX = Luxemburg; LVA = Lettland; MAC = Macao; MEX = Mexiko; $\mathrm{JOR}=$ Jordanien; JPN = Japan; KGZ = Kirgisistan; KOR = Korea; LIE = Liechtenstein; $\mathrm{LTU}=$ Litauen; $\mathrm{LUX}=\mathrm{Luxemburg} ; \mathrm{LVA}=\mathrm{Lettland} ; \mathrm{MAC}=\mathrm{Macao} ; \mathrm{ME}$
$\mathrm{MNE}=$ Montenegro; NLD = Niederlande; $\mathrm{NOR}=$ Norwegen; NZL = Neuseeland; $\mathrm{PAN}=$ Panama; PER = Peru; POL = Polen; PRT = Portugal; $\mathrm{QAT}=\mathrm{Katar} ;$ $\mathrm{MNE}=$ = Montenegro; NLD = Niederlande; $\mathrm{NOR}=$ Norwegen; NZL = Neuseeland; $\mathrm{PAN}=$ Panama; $\mathrm{PER}=\mathrm{P}$
$\mathrm{OCN}=$ Shanghai; $\mathrm{ROU}$ = Rumänien; RUS = Russland; SGP = Singapur; SRB = Serbien; SVK = Slowakei.

Quelle: OECD Pisa-Datenbank 2009; Berechnungen der Autoren.

dest teilweise auf strukturelle Merkmale von Bildungssystemen zurückführen (Allmendinger 1999; Allmendinger/ Leibfried 2003, S. 63). Individuelle Bildungsarmut wird über die vorhandenen Bildungszertifikate oder die Messung der Kompetenzen einer Person operationalisiert. Zugleich wird, der klassischen Armutsforschung folgend, zwischen absoluter und relationaler Bildungsarmut unterschieden. Ein Indikator für absolute Armut von Bildungszertifikaten ist etwa in Deutschland das Fehlen eines Hauptschulabschlusses. Armut in Bezug auf Kompetenzen lässt sich mithilfe großer Schulleistungstests wie der PISA-Studie auch international vergleichend untersuchen. Allmendinger und Leibfried definieren absolute Kompetenzarmut als Nicht-Erreichen der untersten Stufe der PISA-Kompetenzskala, was gleichgesetzt werden kann mit einem „funktionalen Analphabetismus“ (Allmendinger/Leibfried 2003, S.67; Grotlüschen/Riekmann 2012). Wir verwenden im Folgenden eine breitere Definition von kompetenzbezogener Bildungsarmut, die auch die sogenannte Risikogruppe von Schülern einschließt, die lediglich die erste Kompetenzstufe erreicht (Solga 2011, S. 416; Baumert/Maaz 2010, S. 163f.). Diese Schüler verfügen am Ende ihrer Pflichtschulzeit nur über elementare Lesefähigkeiten, sie können lediglich offensichtliche Zusammenhänge zwischen dem Gelesenen und allgemein bekanntem Alltagswissen herstellen, der Inhalt kann jedoch nicht weiter „verarbeitet" werden. ${ }^{1}$ Diesen Definitionen entsprechend waren in PISA 2009 0,8 \% der deutschen 15-Jährigen funktionale Analphabeten und 17,7 \% bildungsarm (OECD 2010a, S. 194). Zur Messung relativer Kompetenzarmut wird in der Regel auf den Anteil derjenigen Schüler verwiesen, deren Kompetenzen mehr als eine Standardabweichung unterhalb des Mittelwerts liegen (Solga 2011, S. 416). ${ }^{2}$

Diese Messung von Bildungsarmut ist damit zunächst nur auf ein bestimmtes Kompetenzniveau am Ende der Pflichtschulzeit ausgerichtet. Längsschnittstudien, in denen die postsekundären Bildungs- und Arbeitsmarktkarrieren von PISA-Teilnehmern erhoben wurden, zeigten allerdings, dass die Performanz auf einem bestimmten Kompetenzniveau eng mit dem späteren Bildungs- und Arbeitsmarkterfolg zusammenhängt. In Kanada ${ }^{3}$ waren Schüler, die im Alter von 15 unterhalb der Lese-Kompetenzstufe 3 abschnitten, zehn Jahre später nur halb so oft in einem postsekundären Bildungsprogramm eingeschrieben wie Schüler, die

1 Auf der PISA-Kompetenzskala bedeutet das für den Bereich Lesen, dass ein Schüler höchstens 407 Punkte erreicht. Damit zeigt dieser Schüler Kompetenzen, die im Vergleich zum OECD-Durchschnitt mindestens um den Lernstand von 2,5 Schuljahren zurückliegen. In Deutschland waren zwei Drittel der in PISA 2009 getesteten Schüler in der 9. Klassenstufe und haben im Schnitt 489 Punkte erreicht. Das heißt, dass bildungsarme 15-jährige Schüler in Deutschland höchstens auf dem Leistungsstand der 7. Klassenstufe sind.

2 Der Anteil relativer Kompetenzarmut ist in Deutschland mit knapp $12 \%$ niedriger als die absolute Kompetenzarmut (eigene Berechnungen mit PISA 2009).

3 Kanada war nicht das einzige Land, das nach den ersten PISA-Studien versucht hat, die Bildungskarrieren von teilnehmenden Schülern im Längsschnitt zu verfolgen. Auch Deutschland hat kürzere Längsschnitte implementiert, die sich jedoch für die Untersuchung von Bildungsarmut weniger eignen, da Schüler ein Jahr nach dem ersten Test ein zweites Mal in der 10. Klassenstufe getestet wurden und somit ein großerTeil der Hauptschüler aus der Untersuchung herausfiel. Ein in Dänemark implementierter Längsschnitt zeigte, dass vier Jahre nach dem Test nur $4 \%$ der lesestarken Schüler nicht eine weiterführende Bildungskarriere eingeschlagen hatten, aber $17 \%$ derjenigen, die unter die erste Kompetenzstufe gefallen waren, keinen erfolgreichen Bildungsabschluss vorweisen konnten (Jensen/Anderson 2006). 
Kompetenzniveau 4 oder 5 erreicht hatten. Nur $10 \%$ der Schüler mit Kompetenzstufe 3 oder darunter hatten zehn Jahre später einen Universitätsabschluss, während mehr als $45 \%$ der „High Performer“ ein solches Zertifikat erworben hatten. Das Einkommen der High Performer lag zudem trotz kürzerer Berufserfahrung mehr als $10 \%$ über dem der „Low Performer“ (Shipley/Gluzynski 2011). Es kann somit angenommen werden, dass die kompetenzbezogene Bildungsarmut im Alter von 15 Jahren ein Risikofaktor für zertifikatsbezogene Bildungsarmut und späteren Arbeitsmarkterfolg einer Person ist.

Das Ausmaß absoluter kompetenzbezogener Bildungsarmut, wie es etwa mit der PISA-Studie messbar ist, unterscheidet sich erheblich zwischen verschiedenen Ländern. Abbildung 1 zeigt, dass in Shanghai weniger als $5 \%$ der getesteten 15-Jährigen nicht die zweite Kompetenzstufe erreichen, während in Aserbaidschan und Kirgistan weniger als $30 \%$ der Schüler nicht als „,bildungsarm“ einzustufen sind.

\section{Theoretische Erklärungen der Entstehung von Bildungsarmut}

Wie können die Entstehung von Bildungsarmut und die Unterschiede zwischen verschiedenen Ländern im Grad der Bildungsarmut theoretisch erklärt werden? Da es sich um ein relativ neues Konzept handelt, liegen bisher keine eigenständigen Ansätze vor, vielmehr wird Bildungsarmut in der Regel als eine extreme Ausprägung von (sozialer) Bildungsungleichheit begriffen. ${ }^{4}$ Innerhalb der soziologischen Bildungs- und Ungleichheitsforschung haben sich verschiedene Theorieansätze zur Erklärung ungleicher Bildungsleistungen entwickelt, die auf unterschiedlichen Ebenen ansetzen. Neben der konflikttheoretischen Perspektive (Bourdieu/Passeron 1964), Theorien „kultureller Reproduktion" (Goldthorpe 1996) oder der Signaling-Theorie (Spence 1974) haben sich in der empirischen Forschung rational-handlungstheoretische Erklärungen etabliert, die Bildungsungleichheit als eine Folge von schichtspezifisch variierenden Leistungen (primäre Effekte) und von zwischen Schichten variierenden Bildungsentscheidungen (sekundäre Effekte) konzipieren (Boudon 1974). Die primären Effekte ergeben sich aus schichtspezifischen Sozialisationsprozessen, aus unterschiedlichen Lernvoraussetzungen und Gelegenheiten und daraus resultierenden schlechteren Leistungen unterer Schichten. Die sekundären Effekte sind Folgen der schichtspezifischen Abwägungen von Kosten und Nutzen verschiedener Bildungsalternativen. Für höhere Schichten droht Statusverlust, wenn nicht bestimmte Bildungsinvestitionen (etwa in Form höherer Bildungsgänge) getätigt werden. Bildungsarmut kann in einer solchen Perspektive als Ergebnis einer wenig förderlichen Sozialisation in Elternhaus und Schule einhergehend mit ausbleibenden oder gescheiterten individuellen Investitionen in
Bildung verstanden werden. Aus dieser Perspektive wird deutlich, dass Bildungsarmut dazu tendiert, sich intergenerational zu verfestigen, wenn nicht starke (kostensenkende) Anreize für weiterführende Bildungsentscheidungen bestehen.

Mit dem Ziel der Erklärung von Unterschieden im Grad der sozialen Bildungsungleichheit zwischen Ländern haben Robert Erikson und Jan O. Jonsson (1996) das Modell von Boudon weiterentwickelt und formalisiert. Sie gehen davon aus, dass die primären Effekte insoweit „universal“ sind, als dass ihr Entstehungsmechanismus in den meisten Gesellschaften ähnlich verläuft und nur geringfügig politischinstitutionell moderiert werden kann. Sie argumentieren auch, dass die schichtspezifischen Investitionen weniger aufgrund „bildungsferner“ Normen zustande kommen als aufgrund von Unterschieden in tatsächlichen Kosten, Erträgen und Wahrscheinlichkeiten von Bildungsinvestitionen, die aus unterschiedlich verteilten familiären Ressourcen resultieren. Der Einfluss der familiären Ressourcen auf die Einschätzung der Entscheidungsparameter wird jedoch durch das Bildungssystem moderiert. Erikson und Jonsson nennen als relevante Bildungssystemmerkmale unter anderem die Länge der unterschiedlichen Bildungsgänge, Regelungen des Übergangs zu weiterführenden Bildungsgängen und Schulgebühren (ebd., S. 33).

Begreift man Bildungsarmut als Folge einer mangelnden Anregung und Förderung im Sozialisationsprozess und als Ergebnis ausbleibender oder gescheiterter Bildungsinvestitionen, können mit diesen Modellen Brückenannahmen formuliert werden, die angeben, aufgrund welcher individueller und institutioneller Bedingungen Investitionen zur Vermeidung von Bildungsarmut ausbleiben. Fraglich ist jedoch, ob die Analyse der Bildungsarmut nicht einen Grenzfall der Anwendbarkeit dieser Erklärungen darstellt, wenn Bildungsarmut etwa mit der Fähigkeit zur Abwägung, zur Kalkulation und zur längerfristigen Planung korreliert ist, da untere Schichten häufig kürzere zeitliche Orientierungen aufweisen (Tismer 1985; Schunck/Rogge 2010). Zudem vernachlässigen die werterwartungstheoretischen Modellierungen die Effekte, die sich aus Zuschreibungs- und Identitätsbildungsprozessen ergeben (Solga 2011, S. 425f.). Negative Etikettierungen während der Schulkarriere und die Konfrontation mit pessimistischen Lehrererwartungen können sich in der Selbstwahrnehmung niederschlagen und zu Rückzugstendenzen führen. Die werterwartungstheoretische Perspektive (u. a. Esser 1999, S. 266ff.) ermöglicht aber die Berücksichtigung solcher Prozesse, etwa in Form geringerer wahrgenommener Realisierungswahrscheinlichkeiten für weiterführende Bildungsentscheidungen bei unteren Schichten.

4 Zur Diskussion der unterschiedlichen theoretischen Implikationen von Bildungsarmut und Bildungsungleichheit vgl. Lohmann/Ferger 2014. 


\section{Wie das Bildungssystem die Entstehung von Bildungsarmut beeinflusst}

In den oben diskutierten theoretischen Ansätzen wurde schon auf mögliche Zusammenhänge zwischen individuellem Bildungserfolg und institutionellen Merkmalen verwiesen. Folgt man der Annahme von Erikson und Jonsson (1996), dass die primären Effekte nicht in der Form moderierbar sind, als dass sie für die deutlichen Unterschiede zwischen Ländern im Grad der Bildungsungleichheit verantwortlich sein können, müssen folglich die Entscheidungsmechanismen ausschlaggebend sein. Welche Merkmale von Bildungssystemen beeinflussen aber die Bildungsentscheidungen und damit die Produktion von Bildungsarmut? Allmendinger (1989) unterscheidet Bildungssysteme anhand ihrer Stratifizierung und Standardisierung. Stratifizierung bezieht sich auf die Differenzierung in verschiedene Schulformen und -curricula (etwa akademischer oder berufsbildender Art). Stratifizierte Schulsysteme variieren in Hinblick auf das Alter, ab welchem die Schüler in verschiedene Schulformen oder Klassen differenziert werden, die Anzahl der verschiedenen Schulformen, die Durchlässigkeit zwischen diesen oder die Handhabung von Versetzungen. Standardisierung bezieht sich auf die Vereinheitlichung der Bildungsstandards und -organisation innerhalb eines Landes (etwa in Bezug auf Lehrerausbildung, Curricula, Prüfungen und Finanzierung). Mit der Standardisierung hängt auch der Grad der Zentralisierung durch eine nationale oder föderale Behörde und die Art und Weise privater Bildungsorganisation zusammen.

Wie können nun diese beiden Dimensionen von Bildungssystemen die Ausprägungen von Bildungsungleichheit und Bildungsarmut in einem Land beeinflussen? Je stratifizierter ein Land ist, desto häufiger und komplexer sind die Entscheidungen, die beim Durchlaufen der Schullaufbahn getroffen werden müssen. Daher steigt die Bedeutung von strategischem Wissen, von langfristigen Zeitperspektiven und von Lehrererwartungen und deren Übergangsempfehlungen. Wenn Eltern keine Erfahrungen mit weiterführenden Bildungsgängen gemacht haben, werden sie dazu tendieren, die Realisierungswahrscheinlichkeiten weiterführender Bildungsübergänge zu unterschätzen, somit kommt es eher zu einer intergenerationalen Verfestigung von Bildungsarmut. Wenn Schüler in leistungshomogenen Klassen unterrichtet werden, können sich negative Schulerfahrungen kollektiv verfestigen und zur Norm werden - Leistungsorientierung kann als Abweichung erscheinen und durch soziale Sanktionen „bestraft" werden. Insbesondere, wenn Übergangsentscheidungen $\mathrm{zu}$ frühen Zeitpunkten getroffen werden müssen, besteht das Risiko, dass sich die tatsächlichen Fähigkeiten eines Kindes noch nicht entfaltet haben, sodass Lehrerempfehlungen durch Zuschreibungen und pessimistische Leistungserwartungen verzerrt sein können.
In Bezug auf die Standardisierung gilt für Bildungsarmut, dass eine ausgeprägte Standardisierung durch Zurechenbarkeit (Accountability) und gleichzeitigen Wettbewerb von Schulen die Anreize erhöht, auch für lernschwächere Schüler förderliche Bedingungen zu schaffen, um die Leistungen aller anzuheben. Eine ausgeprägte institutionelle Privatisierung von Schulsystemen hat hingegen oft ähnliche Effekte wie die Stratifizierung, da besser gestellte Schichten mehr Geld für Schulgeld aufbringen und Schulen mit besseren Ressourcen und Lernbedingungen für ihre Kinder wählen können.

Empirisch wurde der Einfluss von Bildungssystemen überwiegend mit Blick auf das durchschnittliche Kompetenzniveau und den Einfluss des sozioökonomischen Status sowie auf mögliche „Trade-Offs“ zwischen höheren Leistungen und höherer Ungleichheit untersucht (van de Werfhorst/ Mijs 2010). Einflüsse auf kompetenzbezogene Bildungsarmut wurden bislang kaum analysiert. Für die Stratifizierung von Bildungssystemen hat sich gezeigt, dass Schüler höhere Leistungen in nicht-stratifizierten Systemen erbringen. Der Effekt des sozioökonomischen Status ist zudem weniger stark ausgeprägt in Gesamtschulsystemen als in stratifizierten Systemen (Ammermüller 2005; Dronkers et al. 2012; Buchmann/Hannum 2001; Gorard/Smith 2004; Pfeffer 2008). Für die Standardisierung zeigen sich positive Effekte in autonomeren $^{5}$ Schulsystemen, die ein effizientes System der Zurechenbarkeit etabliert haben (Wößmann et al. 2009; Montt 2010; Hanushek et al. 2011). Privatisierung führt zu höheren Leistungen, wenn institutionelle Privatisierung mit geringer finanzieller Privatisierung einhergeht (Wößmann 2006).

Den dargestellten theoretischen Annahmen und empirischen Befunden entsprechend erwarten wir

- höhere Risiken individueller Bildungsarmut in stratifizierten Schulsystemen (Hypothese H 1).

- Für die Standardisierung erwarten wir geringere Bildungsarmut in autonomeren Systemen, in denen jedoch die Zurechenbarkeit der Schulen ausgeprägt ist (H 2).

- Für die Privatisierung erwarten wir negative Effekte auf die Bildungsarmut, wenn eine starke institutionelle Privatisierung mit einer geringen finanziellen Privatisierung einhergeht (H 3).

\section{Daten und Methode}

Die OECD-PISA-Studie als bisher größte internationale Schulleistungsuntersuchung ermöglicht die Analyse einer

5 Dies bezieht sich auf Autonomiedimensionen, die Anreize für opportunistisches Verhalten setzen, also etwa finanzielle Regulierungen oder das Arbeitspensum betreffen (Wößmann 2005, S. 147). 
Reihe von Einflussfaktoren auf das individuelle Risiko für kompetenzbezogene Bildungsarmut. Für unsere Analysen greifen wir auf die Daten der 2009er-Welle zurück, die etwa 470.000 befragte Schüler in insgesamt 65 Teilnehmerstaaten umfasst (OECD 2010a). Die abhängige Variable „Bildungsarmut" wird durch eine dichotome Variable auf der Individualebene gebildet, die den Wert 1 annimmt, wenn eine Schülerin/ein Schüler Leistungen unterhalb der zweiten Kompetenzstufe im Bereich „Lesen“ zeigte. ${ }^{6}$

Um die Stratifizierung von Schulsystemen zu messen, kann auf die Anzahl der verschiedenen Bildungsgänge im sekundären Schulbereich zurückgegriffen werden. Wir verwenden zwei Dummyvariablen für mittlere (zwei oder drei Bildungsgänge) und hohe Stratifizierung (vier oder mehr verschiedene Schulformen). ${ }^{7}$ Zudem wird das Alter der ersten Differenzierung als Indikator herangezogen. Wir messen außerdem die Leistungsgruppierung an Schulen über die Anteile 15-jähriger Schüler in einem Land, die Schulen besuchen, an denen in einigen bzw. allen Fächern nach Leistung getrennt unterrichtet wird. ${ }^{8}$

Für die Messung der Standardisierung betrachten wir Merkmale der Schulautonomie, Zurechenbarkeit und Privatisierung. Für die Analysen wird die Autonomie der Schule mit drei Dummy-Variablen gemessen, die angeben, ob eine Schule Verantwortlichkeit im Hinblick auf das Angebot an Fächern und Kursen („curriculare Autonomie“), die Auswahl des Lehrpersonals („Personalautonomie“) oder die Verwaltung des Schulbudgets („finanzielle Autonomie“) hat. ${ }^{9}$ Diese drei Dummy-Variablen wurden auf Länderebene aggregiert, um den Anteil 15-jähriger Schüler auf autonomen Schulen darzustellen. Die Zurechenbarkeit von Schulen bilden zwei Variablen ab, die den Schüleranteil auf Schulen messen, die Leistungsdaten ihrer Schüler veröffentlichen bzw. zum Vergleich mit anderen Schulen nutzen. ${ }^{10}$ Eine weitere Dummy-Variable gibt an, ob in einem Bildungssystem standardisierte Schulausgangstests durchge- führt werden. ${ }^{11}$ Die Privatisierung von Schulsystemen wird über den Anteil 15-jähriger Schüler auf Privatschulen gemessen. ${ }^{12}$ Die finanzielle Privatisierung wird durch eine Variable abgebildet, die den prozentualen Anteil öffentlicher Finanzierung der Schulen angibt. ${ }^{13}$

Um die Kontexteffekte der Bildungssystemmerkmale von Kompositionseffekten durch unterschiedliche Zusammensetzungen der Schülerschaften zu isolieren, kontrollieren wir auf der Individualebene den Migrantenstatus mithilfe zweier Dummy-Variablen für die erste (nicht im Aufnahmeland geborene) und zweite (im Aufnahmeland geborene) Generation. Den soziokulturellen Status der Familie messen wir mithilfe des „Index of Economic, Social and Cultural Status - ESCS“ (OECD 2005, S. 316f.). Ein wichtiger Faktor für die Entstehung von Bildungsarmut ist der Sprachgebrauch, den wir durch eine dichotome Variable kontrollieren, die den Wert 1 annimmt, sofern zuhause überwiegend die Herkunftssprache gesprochen wird. $\mathrm{Zu}$ dem kontrollieren wir das Geschlecht mithilfe einer Dummy-Variablen für Mädchen und die Familienstruktur mittels einer Dummy-Variablen, die den Wert 1 annimmt, wenn der Schüler mit nur einem Elternteil im Haushalt lebt.

Für die Modellschätzung wurden alle metrischen Variablen um den Gesamtmittelwert zentriert und Fälle mit fehlenden Werten wurden aus der Analyse ausgeschlossen, wodurch der Anteil bildungsarmer Schüler leicht unterschätzt wird. ${ }^{14}$

Es ergibt sich folgende Gleichung:

$$
\begin{aligned}
& \text { logit (Bildungsarmut })_{i j k}=\beta_{0 j k}+\beta \mathbf{X}_{i} \\
& \beta_{0 j k}=\beta_{0}+\gamma \mathbf{Z}_{k}+v_{0 k}+u_{0 j k}
\end{aligned}
$$

wobei $\mathbf{X}$ die Variablen der Individualebene darstellt, $\mathbf{Z}$ die Variablen des Bildungssystems und die Subskripte $i$ für die Schüler, $j$ für die Schulen und $k$ für die Länder stehen.
6 In PISA wie in anderen Schulleistungsstudien werden die auf Basis von Item Response Theory-Modellen ermittelten Kompetenzscores als „plausible Werte“ (Adams/Wu 2002) ausgegeben, die präsentierten Modelle wurden jeweils mit allen fünf plausiblen Werten (PV1READ bis PV5READ) als abhängigen Variablen berechnet, die angegebenen Koeffizienten stellen die Mittelwerte aus den fünf Modellen dar, das Signifikanzniveau wurde auf Basis des für die Varianz über alle fünf Modelle korrigierten Standardfehlers berechnet (vgl. OECD 2005, S. 98).

7 Quelle: OECD 2010b Annex B1: Results for countries and economies, Table IV.3.2a.

8 Quelle: OECD 2010b Annex B1: Results for countries and economies, Table IV.3.4.

9 Quelle: Eigene Berechnungen mit PISA 2009: Curriculare Autonomie liegt vor, wenn für die Variablen SC24QI1 bis SC24QI15 der Wert 1 vorliegt, finanzielle Autonomie wenn die Variablen SC240e1 bis SC240e5 den Wert 1 annehmen und Personalautonomie, wenn die Variablen SC24Qa1 bis
SC24Qa5 den Wert 1 anzeigen (basiert auf Frage 24 des Schulleiterfragebogens).

10 Quelle: OECD 2010b Annex B1: Results for countries and economies, Table IV.3.13.

11 Quelle: OECD 2010b Annex B1: Results for countries and economies, Table IV.3.11. Für die Dummy-Variable wurden Werte kleiner 0.5 als " 0 " kodiert, Werte größer 0.5 als " 1 ".

12 Quelle: Eigene Berechnungen mit PISA 2009: Aggregation einer Dummy-Variable, die den Wert 1 annimmt, wenn die Variable SC02O01=2 ist (Frage 2 im Schulleiterfragebogen).

13 Quelle: Eigene Berechnungen mit PISA 2009, Aggregation der Variable SC03001 (Frage 3 im Schulleiterfragebogen).

14 Aufgrund der Datenverfügbarkeit für die Bildungssystemmerkmale können nicht alle 65 PISA-Teilnehmerländer in die Analyse aufgenommen werden, sondern nur 51 Staaten (Abbildung 1). 
Der Term $v_{0 \mathrm{k}}$ bezeichnet den Fehler der Länderebene (den „Ländereffekt“), der Term $u_{0 \mathrm{jk}}$ die schulspezifischen Abweichungen vom "grand mean" $\beta_{0}$.

Anders als in der OLS-Regression ist in der binären logistischen Regression die abhängige Variable dichotom. Das Modell lässt sich dadurch motivieren, dass die beiden Kategorien beobachtete Realisierungen einer latenten stetigen Zufallsvariable darstellen, deren Verteilung der logistischen Dichtefunktion folgt. Die Residualvarianz dieser Verteilung ist auf den Wert $\pi^{2} / 3$ fixiert. Darum kann sie anders als im OLS Modell - nicht durch weitere erklärungskräftige Prädiktoren reduziert werden (Mood 2010). Vielmehr ändern diese Prädiktoren die Skalierung dieser latenten Variablen (Hox 2010, S. 133). Wir interpretieren dieses Modell daher als latente multiple Regression und beschränken uns dabei auf Vorzeichen und Signifikanz der Koeffizienten. Der Anteil der erklärten Varianz kann unverzerrt nur für Modelle ohne unabhängige Variablen auf der Individualebene angegeben werden (siehe für Korrekturverfahren Hox 2010, S. 136). Tabelle 1 gibt einen Überblick über die verwendeten Variablen.

\section{Ergebnisse}

Für die Analyse des Zusammenhangs von Stratifizierung und Bildungsarmut wird zunächst ein „leeres“ Modell geschätzt, das die Zerlegung der Varianz des individuellen Risikos, bildungsarm zu sein, auf die unterschiedlichen Ebenen (Schüler, Schulen, Länder) ermöglicht. Die Intraklassenkorrelation in Modell 1 in Tabelle 2 zeigt, dass $20 \%$ $(1,313 /(1,313+1,963+3,29)=0,2)$ der Varianz der Bildungsarmut auf Unterschiede zwischen Ländern zurückgehen. In den Modellen 2 und 3 wird der Einfluss der Anzahl der Schulformen getestet. Beide Dummyvariablen, ebenso wie das Alter der Differenzierung (Modell 4 und 5), stehen nicht in signifikantem Zusammenhang mit dem individuellen Risiko für Bildungsarmut.

In den Modellen 3 und 5 werden jeweils die unabhängigen Variablen auf der Individualebene aufgenommen. Es zeigt sich, dass die erste Migrantengeneration ein um den Faktor $1,29\left(e^{0,261}\right)$ signifikant höheres Risiko für Bildungsarmut hat. Wenn der sozioökonomische Status (signifikanter negativer Effekt), der Fremdsprachgebrauch (erhöht das Risiko signi-

TABELLE 1

Variablen und Kennwerte, ungewichtete Stichprobenmerkmale

\begin{tabular}{|c|c|c|c|c|c|}
\hline & Minimum & Maximum & Mittelwert & $\begin{array}{c}\text { Standard- } \\
\text { abweichung }\end{array}$ & $\begin{array}{c}\text { Häufigkeit } \\
\text { (Dummy = 1) }\end{array}$ \\
\hline Bildungsarmut & 0 & 1 & 0,26 & & 90464,4 \\
\hline Zweite Generation & 0 & 1 & 0,052 & & 22,571 \\
\hline Index of economic, social and cultural status (ESCS) & $-6,6195$ & 3,5346 & $-0,26$ & 1,130 & \\
\hline Sprachgebrauch: Herkunftssprache & 0 & 1 & 0,113 & & 48,372 \\
\hline \multicolumn{6}{|l|}{ Länderebene $^{b}$} \\
\hline \multicolumn{6}{|l|}{ Stratifizierung (N Länder $=51)$} \\
\hline Hohe Stratifizierung & 0 & 1 & 0,37 & & 19 \\
\hline Mittlere Stratifizierung & 0 & 1 & 0,20 & & 15 \\
\hline Alter der Selektion & 10 & 17 & 14,63 & 1,70 & \\
\hline Fächerbez. Gruppierung & 0,6 & 93,6 & 50,28 & 20,55 & \\
\hline Finanzielle Autonomie & 0,063 & 0,993 & 0,51 & 0,24 & \\
\hline Schulleistungen werden veröffentlicht & 0,6 & 86 & 35,37 & 21,66 & \\
\hline Schulleistungen werden verglichen & 6,5 & 95 & 49,56 & 22,35 & \\
\hline Stand. Ausgangstests & 0 & 1 & 0,609 & & 38 \\
\hline Anteil Privatschüler & 0,001 & 0,960 & 0,193 & 0,212 & \\
\hline Anteil öffentliche Finanzierung & 43,94 & 99,78 & 79,65 & 17,21 & \\
\hline
\end{tabular}

a Fallzahlen beziehen sich auf das nach Ausschluss fehlender Werte für die Schätzung der Modelle in Tabelle 5 verwendete Sample. b Die hier dargestellten Häufigkeiten beziehen sich auf das Ländersample. 
TABELLE 2

Stratifizierung von Bildungssystemen und individuelles Risiko von Bildungsarmut, Ergebnisse der logistischen Mehrebenenregression

\begin{tabular}{|c|c|c|c|c|c|c|c|c|c|}
\hline & (1) & (2) & (3) & (4) & (5) & (6) & (7) & (8) & (9) \\
\hline Intercept & $-1,432^{* * *}$ & $-1,513^{* * *}$ & $-1,217 * * *$ & $-1,380 * * *$ & $-1,113^{* * *}$ & $-1,439 * * *$ & $-1,158 * * *$ & $-1,434 * * *$ & $-1,157 * * *$ \\
\hline Erste Generation & & & $0,261 * * *$ & & $0,269 * * *$ & & $0,271 * * *$ & & $0,270 * * *$ \\
\hline Zweite Generation & & & 0,0337 & & 0,0302 & & 0,0344 & & 0,0331 \\
\hline ESCS & & & $-0,408 * * *$ & & $-0,417 * * *$ & & $-0,419 * * *$ & & $-0,420 * * *$ \\
\hline Mädchen & & & $-0,827 * * *$ & & $-0,848 * * *$ & & $-0,853 * * *$ & & $-0,858 * * *$ \\
\hline Fremdsprache & & & $0,434 * * *$ & & $0,449 * * *$ & & $0,452 * * *$ & & $0,452 * * *$ \\
\hline Alleinerziehend & & & $0,150 * * *$ & & $0,152 * * *$ & & $0,152 * * *$ & & $0,153 * * *$ \\
\hline Mittlere Stratifizierung & & 0,464 & 0,343 & & & & & & \\
\hline Hohe Stratifizierung & & $-0,0748$ & $-0,0718$ & & & & & & \\
\hline Alter der Selektion & & & & 0,0303 & 0,0421 & & & & \\
\hline Fächerbez. Gruppierung & & & & & & $-0,0155^{*}$ & $-0,0121+$ & & \\
\hline Leistungsgruppierung & & & & & & & & $0,0318^{* *}$ & $0,0287^{*}$ \\
\hline \multicolumn{10}{|l|}{ Varianzkomponenten } \\
\hline$\sigma^{2} v 0$ (Land) & $1,313^{* * *}$ & $1,155^{* * *}$ & $1,087 * * *$ & $1,261 * * *$ & $1,158 * * *$ & $1,172^{* * *}$ & $1,104 * * *$ & $1,141 * * *$ & $1,057^{* * *}$ \\
\hline$\sigma^{2} u 0$ (Schule) & $1,963 * * *$ & $1,586 * * *$ & $1,321 * * *$ & $1,787 * * *$ & $1,424 * * *$ & $1,882 * * *$ & $1,467 * * *$ & $1,905^{* * *}$ & $1,475^{* * *}$ \\
\hline$\sigma^{2} e 0$ (Schüler) & $1 * * *$ & $1 * * *$ & 1 & 1 & 1 & $1 * * *$ & $1 * * *$ & $1 * * *$ & $1 * * *$ \\
\hline
\end{tabular}

$+p<0,1,{ }^{*} p<0,05,{ }^{* *} p<0,01,{ }^{* * *} p<0,001$

Quelle: Berechnungen der Autoren

fikant um den Faktor 1,5), das Geschlecht (Mädchen haben ein signifikant geringeres Risiko) und der Familienstand (Kinder Alleinerziehender haben ein um den Faktor 1,16 erhöhtes Risiko) kontrolliert sind, haben Migranten zweiter Generation im Vergleich zu Einheimischen kein signifikant höheres Risiko, unter die zweite Kompetenzstufe zu fallen.

Ein weiterer Indikator für die Stratifizierung von Schulsystemen ist die Praxis der Leistungsgruppierung an Schulen (Modelle 6 bis 9). Für eine fächerbezogene Leistungsgruppierung zeigt sich, dass ein höherer Schüleranteil an Schulen mit einer solchen Form des trackings zu einem geringeren individuellen Risiko für kompetenzbezogene Bildungsarmut führt. Hingegen führt die Leistungsgruppierung in allen Fächern zu einem höheren Risiko von Bildungsarmut (Modell 8 und 9). So beträgt die Wahrscheinlichkeit (berechnet auf Basis von Modell 9) für das durchschnittliche Individuum in einem unterdurchschnittlich ${ }^{15}$ leistungsgruppierten Land bildungsarm zu sein, $13 \%$. Demgegenüber beträgt sie in einem Land mit überdurchschnittlich verbreiteter Leistungsgruppierung $23 \%$. Wenn die Leistungsgruppierung in allen Fächern als einzige unabhängige Variable in das Modell (8) aufgenommen wird, werden $13 \%$ der Varianz der Länderebene erklärt.

Insgesamt zeigt sich, dass, wenn signifikante Effekte für die Stratifizierung gemessen werden, diese die Hypothesen insofern bestätigen, als dass eine höhere Stratifizierung mit einem höheren Risiko für Bildungsarmut einhergeht. Allerdings ist eine fächerbezogene Leistungsgruppierung eher förderlich für die Vermeidung von Bildungsarmut, dies könnte ein Effekt von gezieltem Förderunterricht in bestimmten Fächern sein, der jedoch nicht in vollständiger Leistungssegregation resultiert und die negativen Effekte durch Stigmatisierung und geringe Leistungserwartungen vermeidet.

Für die Analyse der Standardisierung schätzen wir wiederum zunächst ein leeres Modell zur Varianzzerlegung (Modell 1 in Tabelle 3). Hier gehen 23 \% der Gesamtvarianz auf Unterschiede zwischen Ländern zurück. In den Modellen 2 bis 7 werden die Effekte verschiedener Schulautonomie-Dimensionen auf das Risiko, unter die zweite Lesekompetenzstufe zu fallen, geschätzt. Die Modelle 2 und 3 zeigen, dass curriculare Autonomie das Risiko für Bildungsarmut senkt. Die Autonomie (Modell 2) erklärt 15 \% der Varianz auf der Länderebene. Die Personalautonomie (Modelle 4 und 5) steht in schwach signifikantem negativem Zusammenhang mit dem Risiko für Bildungsarmut, ist jedoch nicht mehr signifikant, wenn Individualmerkmale kontrolliert werden. Für die finanzielle Autonomie (Modelle 6 und 7) zeigt sich ein ähnliches Ergebnis, allerdings steigt hier die Signifikanz, wenn die Individualmerkmale kontrolliert werden. Schulautonomie in den Entscheidungen darüber, welche Kurse angeboten werden, hat demnach den stärksten

15 Unter- bzw. überdurchschnittlich ist hier und im Folgenden definiert als eine Standardabweichung unterhalb bzw. oberhalb des Mittelwerts. 
TABELLE 3

Standardisierung von Bildungssystemen und individuelles Risiko von Bildungsarmut, Ergebnisse der logistischen Mehrebenenregression

\begin{tabular}{|c|c|c|c|c|c|c|c|}
\hline & (1) & (2) & (3) & (4) & (5) & (6) & (7) \\
\hline Intercept & $-1,476 * * *$ & $-1,367 * * *$ & $-1,108^{* * *}$ & $-1,351 * * *$ & $-1,109 * * *$ & $-1,400 * * *$ & $-1,132 * * *$ \\
\hline Erste Generation & & & $0,247 * * *$ & & $0,239 * * *$ & & $0,241 * * *$ \\
\hline Zweite Generation & & & 0,0246 & & 0,0247 & & 0,0250 \\
\hline ESCS & & & $-0,421 * * *$ & & $-0,419 * * *$ & & $-0,423 * * *$ \\
\hline Mädchen & & & $-0,834 * * *$ & & $-0,825^{* * *}$ & & $-0,832 * * *$ \\
\hline Fremdsprache & & & $0,498 * * *$ & & $0,473 * * *$ & & $0,476 * * *$ \\
\hline Alleinerziehend & & & $0,134^{* * *}$ & & $0,134 * * *$ & & $0,135^{* * *}$ \\
\hline Curriculare Autonomie & & $-1,669 * *$ & $-1,595 * *$ & & & & \\
\hline Personalautonomie & & & & $-0,949+$ & $-0,699$ & & \\
\hline Finanzielle Autonomie & & & & & & $-1,177+$ & $-1,314^{*}$ \\
\hline \multicolumn{8}{|l|}{ Varianzkomponenten } \\
\hline$\sigma^{2} v 0$ (Land) & $1,563 * * *$ & $1,318 * * *$ & $1,216 * * *$ & $1,389 * * *$ & $1,313^{* * *}$ & $1,371 * * *$ & $1,241 * * *$ \\
\hline$\sigma^{2} u 0$ (Schule) & $1,918 * * *$ & $1,692 * * *$ & $1,416 * * *$ & $1,570 * * *$ & $1,323 * * *$ & $1,617 * * *$ & $1,351 * * *$ \\
\hline$\sigma^{2} e 0$ (Schüler) & $1 * * *$ & $1 * * *$ & $1 * * *$ & 1 & $1 * * *$ & $1 * * *$ & $1 * * *$ \\
\hline
\end{tabular}

$+p<0,1, * p<0,05, * * p<0,01, * * * p<0,001$

Effekt auf die Vermeidung von Bildungsarmut. Erklärbar wäre ein solcher Effekt mit der Möglichkeit von Schulen, bestimmte Förderkurse für schwächere Schüler anzubieten.

Die Zurechenbarkeit von Schulen ist ein Merkmal der Standardisierung von Schulsystemen, die sich in empirischen Arbeiten als positiv für die Chancengerechtigkeit im

ABB. 2

Wahrscheinlichkeit für Bildungsarmut nach sozioökonomischem Status und institutioneller Privatisierung

— unterdurchschnittliche institutionelle Privatisierung

— überdurchschnittliche Privatisierung

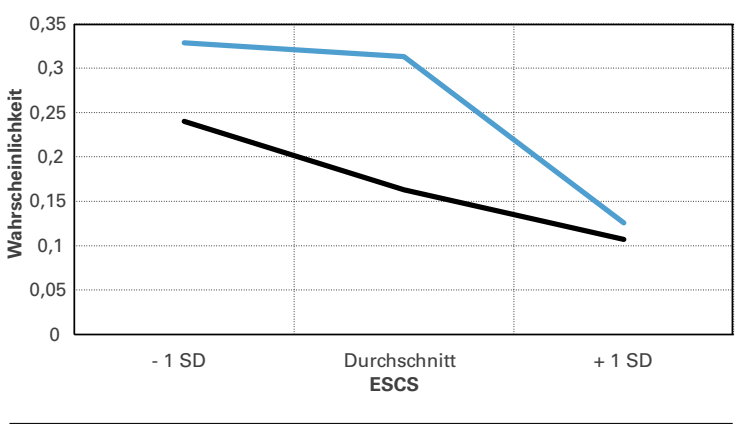

Quelle: OECD Pisa-Datenbank 2009; Berechnungen der Autoren
Bildungssystem erwiesen hat. Wenn in einem Land ein höherer Anteil von Schülern Schulen besucht, die Leistungsdaten veröffentlichen, geht dies allerdings nicht mit geringeren Risiken für kompetenzbezogene Bildungsarmut einher; im Gegenteil, das individuelle Risiko ist bei vergleichbarem familiären Hintergrund (Modell 2 in Tabelle 4) sogar erhöht. Ebenso steht ein höherer Schüleranteil an Schulen, die Leistungsdaten zum Vergleich mit anderen Schulen nutzen, mit einem höheren individuellen Risiko für Bildungsarmut in Zusammenhang (Modelle 3 und 4). Lediglich die Standardisierung durch externe Schulausgangstests (Modell 5) geht mit einem geringeren Risiko, unter die zweite Kompetenzstufe im Lesen zu fallen, einher (schwach signifikant unter Kontrolle der Individualmerkmale in Modell 6).

Schließlich ist ein weiteres wichtiges Merkmal von Schulsystemen der Grad ihrer Privatisierung. Ein höherer Anteil von Schülern auf Privatschulen in einem Land steht nicht in signifikantem Zusammenhang mit dem Risiko für Bildungsarmut (Modelle 1 und 2 in Tabelle 5). Allerdings interagiert der Privatschüleranteil signifikant mit dem Effekt des sozioökonomischen Status (Abbildung 2).

Ein direkter, signifikanter Effekt auf die Bildungsarmut zeigt sich jedoch für die finanzielle Privatisierung (Modell 3): Je höher der Anteil der öffentlichen Finanzierung an Schulen in einem Land, desto geringer ist das individuelle Risiko, unterhalb der zweiten Kompetenzstufe in Lesen abzuschneiden.

Zusätzlich zeigt sich in diesen Ländern ein stärkerer protektiver Effekt des sozioökonomischen Status auf das Risiko, bildungsarm zu sein (Interaktionseffekt in Modell 4). 
Zurechenbarkeit in Bildungssystemen und individuelles Risiko von Bildungsarmut, Ergebnisse der logistischen Mehrebenenregression

\begin{tabular}{|c|c|c|c|c|c|c|}
\hline & (1) & (2) & (3) & (4) & (5) & (6) \\
\hline Intercept & $-1,464 * * *$ & $-1,185^{* * *}$ & $-1,449 * * *$ & $-1,181 * * *$ & $-0,879 * *$ & $-0,707^{*}$ \\
\hline Erste Generation & & $0,241 * * *$ & & $0,241 * * *$ & & $0,236 * * *$ \\
\hline Zweite Generation & & 0,0210 & & 0,0229 & & 0,0224 \\
\hline ESCS & & $-0,425 * * *$ & & $-0,423^{* * *}$ & & $-0,411 * * *$ \\
\hline Mädchen & & $-0,838 * * *$ & & $-0,833^{* * *}$ & & $-0,814 * * *$ \\
\hline Fremdsprache & & $0,490 * * *$ & & $0,481 * * *$ & & $0,484 * * *$ \\
\hline Alleinerziehend & & $0,134 * * *$ & & $0,134 * * *$ & & $0,132 * * *$ \\
\hline Veröffentlichen & 0,0107 & $0,0122+$ & & & & \\
\hline Vergleichen & & & $0,0145^{*}$ & $0,0139 *$ & & \\
\hline Stand. Ausgangstests & & & & & $-0,770^{*}$ & $-0,655+$ \\
\hline \multicolumn{7}{|l|}{ Varianzkomponenten } \\
\hline$\sigma^{2} v 0$ (Land) & $1,425 * * *$ & $1,288 * * *$ & $1,368 * * *$ & $1,258 * * *$ & $1,340 * * *$ & $1,272 * * *$ \\
\hline \multicolumn{7}{|l|}{$\sigma^{2} v E s c s$} \\
\hline$\sigma^{2} u 0$ (Schule) & $1,820 * * *$ & $1,427 * * *$ & $1,699 * * *$ & $1,382 * * *$ & $1,536 * * *$ & $1,335 * * *$ \\
\hline$\sigma^{2} e 0$ (Schüler) & $1 * * *$ & 1 & $1 * * *$ & $1 * * *$ & 1 & 1 \\
\hline
\end{tabular}

TABELLE 5

Privatisierung von Bildungssystemen und individuelles Risiko von Bildungsarmut, Ergebnisse der logistischen Mehrebenenregression

\begin{tabular}{|c|c|c|c|c|c|}
\hline & (1) & (2) & (3) & (4) & (5) \\
\hline Intercept & $-1,426 * * *$ & $-1,141 * * *$ & $-0,922 * * *$ & $-0,775 * * *$ & $-0,855 * * *$ \\
\hline Erste Generation & & $0,241 * * *$ & & $0,175 * * *$ & $0,181 * * *$ \\
\hline Zweite Generation & & 0,0091 & & 0,00429 & 0,00541 \\
\hline ESCS & & $-0,488 * * *$ & & $-0,355^{* * *}$ & $-0,367 * * *$ \\
\hline Mädchen & & $-0,837 * * *$ & & $-0,633 * * *$ & $-0,642 * * *$ \\
\hline Fremdsprache & & $0,485^{* * *}$ & & $0,360 * * *$ & $0,366 * * *$ \\
\hline Alleinerziehend & & $0,119 * * *$ & & $0,0943 * * *$ & $0,0958 * * *$ \\
\hline Anteil Privatschüler & $-0,424$ & $-0,721$ & & & $-0,638$ \\
\hline Ant. Privatschüler*ESCS & & $0,271 * *$ & & & \\
\hline Öffentliche Finanzierung & & & $-0,0181^{*}$ & $-0,0135+$ & $-0,0165^{*}$ \\
\hline Öff. Finanz*ESCS & & & & $-0,00275+$ & \\
\hline Anteil Privats. ${ }^{*}$ Öff. Finanz. & & & & & $-0,0709$ \\
\hline \multicolumn{6}{|l|}{ Varianzkomponenten } \\
\hline$\sigma^{2} v 0$ (Land) & $1,460 * * *$ & $1,307 * * *$ & $0,799 * * *$ & $0,742 * * *$ & $0,631 * * *$ \\
\hline$\sigma^{2} v E s c s$ & & $0,024 * *$ & & $0,0237 * * *$ & $0,0229 * * *$ \\
\hline$\sigma^{2} u 0$ (Schule) & $1,809 * * *$ & $1,42 * * *$ & $0,889 * * *$ & $0,682^{* * *}$ & $0,720 * * *$ \\
\hline$\overline{\sigma^{2} e 0 ~(S c h u ̈ l e r) ~}$ & 1 & 1 & $1 * * *$ & $1 * * *$ & 1 \\
\hline
\end{tabular}

$+p<0,1, * p<0,05,{ }^{* *} p<0,01, * * * p<0,001$

Quelle: Berechnungen der Autoren. 


\section{Wahrscheinlichkeit für Bildungsarmut nach sozioökonomischem Status und finanzieller Privatisierung}

— unterdurchschnittliche öffentliche Finanzierung

— überdurchschnittliche öffentliche Finanzierung

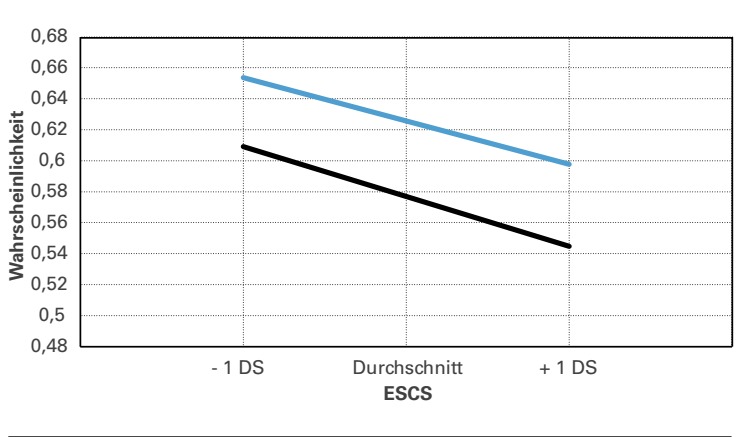

Quelle: OECD Pisa-Datenbank 2009; Berechnungen der Autoren.

Abbildung 3 zeigt, dass die Kurve, die die Wahrscheinlichkeit für Bildungsarmut in Abhängigkeit des sozialen Status abbildet, in Ländern mit überdurchschnittlicher öffentlicher Finanzierung etwas steiler verläuft. Die Effekte der institutionellen Privatisierung und der finanziellen Privatisierung interagieren nicht signifikant miteinander, wie in den $\mathrm{Hy}$ -

TABELLE 6

Standardisierung und Stratifizierung von Bildungssystemen und individuelles Risiko von Bildungsarmut, Ergebnisse der logistischen Mehrebenenregression

\begin{tabular}{|c|c|c|c|}
\hline & (1) & (2) & (3) \\
\hline Intercept & $-1,372 * * *$ & $-0,899 * * *$ & $-0,771 * * *$ \\
\hline Erste Generation & & & $0,198 * * *$ \\
\hline Zweite Generation & & & 0,0104 \\
\hline ESCS & & & $-0,334 * * *$ \\
\hline Mädchen & & & $-0,653 * * *$ \\
\hline Fremdsprache & & & $0,337 * * *$ \\
\hline Alleinerziehend & & & $0,116 * * *$ \\
\hline Curriculare Autonomie & & $-0,994^{* *}$ & $-1,033^{* *}$ \\
\hline Öffentliche Finanzierung & & $-0,0110+$ & $-0,00550$ \\
\hline Leistungsgruppierung & & $0,0275^{* * *}$ & $0,0271 * * *$ \\
\hline \multicolumn{4}{|l|}{ Varianzkomponenten } \\
\hline$\sigma^{2} v 0$ (Land) & $1,563^{* * *}$ & $1,318 * * *$ & $1,216 * * *$ \\
\hline$\sigma^{2} u 0$ (Schule) & $1,918 * * *$ & $1,692 * * *$ & $1,416 * * *$ \\
\hline$\sigma^{2} e 0$ (Schüler) & $1 * * *$ & $1 * * *$ & $1 * * *$ \\
\hline McKelvey \& Zavoina R² & , & 0,049 & 0,19 \\
\hline
\end{tabular}

$+p<0,1,{ }^{*} p<0,05,{ }^{* *} p<0,01,{ }^{* * *} p<0,001$

Quelle: Berechnungen der Autoren. pothesen angenommen. Insgesamt zeigt sich für die Standardisierung, dass Schulautonomie und die Existenz von standardisierten Schulausgangstests das Risiko für Bildungsarmut verringern. Zudem zeigt sich, dass finanzielle Privatisierung eher mit höherer Bildungsarmut einhergeht.

In einem letzten Modell sollen nun die ermittelten einflussreichen Merkmale von Standardisierung und Stratifizierung (curriculare Autonomie, öffentliche Finanzierung und Leistungsgruppierung in allen Fächern) gemeinsam betrachtet werden (Tabelle 6). Die Effekte bleiben in Modell 1 robust, allerdings ist der Effekt der öffentlichen Finanzierung hier nur schwach signifikant. Durch die drei Indikatoren werden immerhin 45 \% der Varianz auf der Länderebene erklärt, jedoch insgesamt nur knapp $5 \%$ der Gesamtvarianz (basierend auf dem McKelvey und Zavoina $\mathrm{R}^{2}$ ). Im „vollen“ Modell 2 ist die öffentliche Finanzierung nicht mehr signifikant. Hier werden schließlich knapp $20 \%$ der Gesamtvarianz des Risikos für kompetenzbezogene Bildungsarmut erklärt.

\section{Zusammenfassung und Diskussion}

In modernen wissensbasierten Ökonomien stellt Bildungsarmut eine zunehmend wichtige sozialpolitische Kategorie dar. Auf der Individualebene kann Bildungsarmut die Lebenschancen erheblich beeinträchtigen, auf der gesellschaftlichen Ebene drohen hohe soziale und politische Folgekosten. In unserem Beitrag haben wir den Zusammenhang von Stratifizierung und Standardisierung von Bildungssystemen mit dem individuellen Risiko absoluter kompetenzbezogener Bildungsarmut untersucht. Unsere Analysen zeigen, dass die Annahme, eine höhere Stratifizierung des Bildungssystems erhöhe das Risiko für Bildungsarmut, für die Praxis der Leistungsgruppierung in allen Fächern bestätigt werden kann (H1). Wir konnten zeigen, dass Schulautonomie und Standardisierung (externe Schulausgangstests) mit einem geringeren Risiko, unter die zweite Kompetenzstufe zu fallen, in Zusammenhang stehen (H2). Ein höherer Anteil von Schülern, die Privatschulen besuchen, geht nicht mit einem geringeren Risiko für Bildungsarmut einher, jedoch senkt ein höherer Anteil öffentlicher Finanzierung das Risiko für Bildungsarmut (H3).

Dennoch schließen sich weitere Fragen an, die an dieser Stelle noch nicht beantwortet werden können: Gibt es Länder, in denen bestimmte Faktoren weniger oder mehr einflussreich sind, weil sie mit anderen institutionellen Merkmalen interagieren? Gibt es Gruppen von Ländern, die sich hinsichtlich der Merkmale und der Effekte auf Bildungsarmut ähneln? Wie wirkt sich kompetenzbezogene Bildungsarmut im Alter von 15 Jahren auf relative Bildungsarmut und auf zertifikatsbezogene Bildungsarmut und spätere Lebenschancen aus? Bei Analysen der kausalen Bedingungen eines Konstruktes wie Bildungsarmut muss zudem bedacht werden, dass Hinweise auf kausale Effekte mit den 
Querschnittsdaten der PISA-Studie kaum erbracht werden können. Dies steht im Widerspruch zu dem politischen Beratungsanspruch, den die OECD mit der Studie einnimmt. So kann es sein, dass nicht die hier betrachteten institutionellen Faktoren, sondern mit diesen konfundierte, jedoch nicht gemessene Merkmale ursächlich sind. Umso problematischer sind daher bildungspolitische Reformbemühungen einzuschätzen, wenn sie sich allein auf die Ergebnisse von querschnittlichen Assessments stützen. Seit Beginn der PISA-Studien im Jahr 2000 sind in vielen Teilnahmeländern teils umfassende Veränderungen in den Bildungssystemen angestoßen worden. Da umfassende internationale Längsschnittstudien im Bildungsbereich kaum realisierbar sind, sollte sich zukünftige Forschung entsprechend auch auf die Weiterentwicklung geeigneter Verfahren zur Kausalanalyse von wiederholt durchgeführten Querschnittsstudien konzentrieren.

\section{LITERATUR}

Adams, R./Wu, M. (2002): PISA 2000 Technical Report, Paris, http://www.sourceoecd.org/9264199519

Allmendinger, J. (1989): Educational systems and labor market outcomes, in: European Sociological Review 5 (3), S. 231-250

Allmendinger, J. (1999): Bildungsarmut: zur Verschränkung von Bildungs- und Sozialpolitik, in: Soziale Welt 50 (1), S. 35-50

Allmendinger, J./Giesecke, J./Oberschachtsiek, D. (2011): Unzureichende Bildung. Folgekosten für die öffentlichen Haushalte, Gütersloh

Allmendinger, J./Leibfried, S. (2003): Four worlds of competence production, in: Journal of European Social Policy 13 (1), S. 63-81

Ammermüller, A. (2005): Poor background or low returns? Why immigrant stu dents in Germany perform so poorly in PISA, ZEW Discussion Paper 05-18, Mannheim

Bacher, J./Hirtenlehner, H./Kupfer, A. (2010): Politische und soziale Folgen von Bildungsarmut, in: Quenzel, G./Hurrelmann, K. (Hrsg.): Bildungsverlierer Neue Ungleichheiten, Wiesbaden, S. 475-496

Baumert, J./Maaz, K. (2010): Bildungsungleichheit und Bildungsarmut - Der Beitrag von Large-Scale-Assessments, in: Quenzel, G./Hurrelmann, K. (Hrsg.): Bildungsverlierer - Neue Ungleichheiten, Wiesbaden, S. 159-179

Boudon, R. (1974): Education, opportunity, and social inequality: Changing prospects in western society, New York

Bourdieu, P./Passeron, J.-C. (1964) : Les étudiants et leurs études, Paris [u.a.] Buchmann, C./Hannum, E. (2001): Education and stratification in developing countries: A review of theories and research, in: Annual Review of Sociology 27 (1), S. 77-102

Dronkers, J./van der Velden, R./Dunne, A. (2012): Why are migrant students better off in certain types of educational systems or schools than in others?, in: European Educational Research Journal 11 (1), S. $11-44$

Erikson, R./Jonsson, J. O. (1996): Introduction: Explaining class inequality in education. The Swedish test case, in: Erikson, R./Jonsson, J. O. (Hrsg.): Can education be equalized? The Swedish case in comparative perspective, Boulder, Colo., S. 1-63

Esser, H. (1999): Situationslogik und Handeln. Soziologie, Spezielle Grundlagen, Bd. 1, Frankfurt a. M.

Giesecke, J./Ebner, C./Oberschachtsiek (2010): Bildungsarmut und Arbeitsmarktexklusion, in: Quenzel, G./Hurrelmann, K. (Hrsg.): Bildungsverlierer Neue Ungleichheiten, Wiesbaden, S. 421-438

Goldthorpe, J. H. (1996): Class analysis and the reorientation of class theory: The case of persisting differentials in educational attainment, in:The British Journal of Sociology 47 (3), S. $481-506$

Gorard, S./Smith, E. (2004): An international comparison of equity in education systems, in: Comparative Education 40 (1), S. 15-28

Grotlüschen, A./Riekmann, W. (2012): Funktionaler Analphabetismus in Deutschland. Ergebnisse der ersten leo.-Level One Studie, Münster

Hanushek, E. A./Link, S./Wößmann, L. (2011): Does school autonomy make sense everywhere? Panel estimates from PISA, NBER Working Paper (17591), Cambridge, MA

Hox, J. J. (2010): Multilevel analysis. Techniques and applications, New York Jensen, T. P./Andersen, D. (2006): Participants in PISA 2000 - Four years later, in: Mejding, J./Roe, A. (Hrsg.): Northern lights on PISA 2003. A reflection from the Nordic countries, S. 223-235
Lohmann, H./Ferger, F. (2014): Educational poverty in a comparative perspective:Theoretical and empirical implications, SFB 882 Working Paper Series (26), Bielefeld

Ludwig-Mayerhofer, W./Kühn, S. (2010): Bildungsarmut, Exklusion und die Rolle von sozialer Verarmung und Social Illiteracy, in: G. Quenzel/K. Hurrelmann (Hrsg.): Bildungsverlierer - Neue Ungleichheiten, Wiesbaden, S. $137-155$

Montt, G. (2010): Cross-national differences in educational achievement inequality, in: Sociology of Education 84 (1), S. 49-68

Mood, C. (2010): Logistic regression: Why we cannot do what we think we can do, and what we can do about it, in: European Sociological Review 26 (1), S. $67-82$

Organisation for Economic Co-operation and Development (OECD) (2005): PISA 2003 data analysis manual, Paris

Organisation for Economic Co-operation and Development (OECD) (2010a): PISA 2009 results, Vol. I: What students know and can do, Paris Organisation for Economic Co-operation and Development (OECD) (2010b): PISA 2009 results, Vol. IV: What makes a school successful? Resources, policies and practices, Paris

Pfeffer, F.T. (2008): Persistent inequality in educational attainment and its institutional context, in: European Sociological Review 24 (5), S. 543-565 Schunck, R./Rogge, B. (2010): Unemployment and its association with healthrelevant actions: Investigating the role of time perspective with German census data, in: International Journal of Public Health 55 (4), S. 271-278 Shipley, L./Gluzynski, T. (2011): Life-path outcomes at age 25 associated with reading ability at age 15 . Education matters: Insights on education, learning and training in Canada 8 (2),

http://www.statcan.gc.ca/pub/81-004-x/2011002/article/11490-eng.htm

Solga, H. (2005): Ohne Abschluss in die Bildungsgesellschaft. Die Erwerbschancen gering qualifizierter Personen aus soziologischer und ökonomischer Perspektive, Opladen

Solga, H. (2011): Bildungsarmut und Ausbildungslosigkeit in der Bildungs- und Wissensgesellschaft, in: Becker, R. (Hrsg.): Lehrbuch der Bildungssoziologie, Wiesbaden, S. 411-448

Spence, A. M. (1974): Market signaling. Informational transfer in hiring and related screening processes, Cambridge, MA

Tismer, K.-G. (1985): Zeitperspektive und soziale Schichtzugehörigkeit, in: Kölner Zeitschrift für Soziologie und Sozialpsychologie 37 (4), S. 677-697 van de Werfhorst, H. G./Mijs, J. J. B. (2010): Achievement inequality and the institutional structure of educational systems. A comparative perspective, in: Annual Review of Sociology 36 (1), S. 407-428

Wößmann, L. (2005): The effect heterogeneity of central examinations: evidence from TIMSS, TIMSS-Repeat and PISA, in: Education Economics 13 (2), S. $143-169$

Wößmann, L. (2006): Public-private partnership and schooling outcomes across countries, CESifo Working Paper Series (1662)

Wößmann, L./Lüdemann, E./Schuetz, G./West, M. R. (2009): School accountability, autonomy and choice around the world, Northampton, MA

Wößmann, L./Piopiunik, M. (2009): Was unzureichende Bildung kostet. Eine Berechnung der Folgekosten durch entgangenes Wirtschaftswachstum, München

\section{AUTOREN}

JANNA TELTEMANN, Dr. rer. pol. Soziologin, ist Wissenschaftliche Mitarbeiterin am Institut für empirische und angewandte Soziologie (EMPAS) an der Universität Bremen. Arbeitsschwerpunkte: Ethnische Bildungsungleichheit, Bildungspolitik, Empirische Stadtforschung.

janna@uni-bremen.de

MICHAEL WINDZIO, Prof. Dr., Soziologe, ist Direktor des EMPAS und Professor für Migration und Stadtforschung an der Universität Bremen. Arbeitsschwerpunkte: Stadt und Migration, Kriminalität und abweichendes Verhalten, Quantitative Methoden der empirischen Sozialforschung.

mwindzio@empas.uni-bremen.de 